\title{
Development of mannose-anchored thiolated amphotericin B nanocarriers for treatment of visceral leishmaniasis
}

\begin{abstract}
Aim: Our goal was to improve treatment outcomes for visceral leishmaniasis by designing nanocarriers that improve drug biodistribution and half-life. Thus, longacting mannose-anchored thiolated chitosan amphotericin B nanocarrier complexes (MTC AmB) were developed and characterized. Materials \& methods: A mannoseanchored thiolated chitosan nanocarrier was manufactured and characterized. MTC AmB was examined for cytotoxicity, biocompatibility, uptake and antimicrobial activities. Results: MTC AmB was rod shaped with a size of $362 \mathrm{~nm}$. MTC AmB elicited $90 \%$ macrophage viability and 71-fold enhancement in drug uptake compared with native drug. The antileishmanial IC $\mathrm{IC}_{50}$ for MTC AmB was $0.02 \mu \mathrm{g} / \mathrm{ml}$ compared with $0.26 \mu \mathrm{g} / \mathrm{ml}$ for native drug. Conclusion: These studies show that MTC can serve as a platform for clearance of Leishmania in macrophages.
\end{abstract}

First draft submitted: 2 September 2016; Accepted for publication: 26 October 2016; Published online: 23 November 2016

Keywords: amphotericin B • macrophage nanoparticle targeting • mannose receptors - thiolated chitosan • visceral leishmaniasis

Visceral leishmaniasis (VL; kala-azar) remains a common tropical infectious disease. Leishmania amastigotes and promastigotes target mononuclear phagocytes (MP; monocytes and tissue macrophages), and parasite growth is sustained by the cell's microenvironment. Infection remains high in prevalence, morbidity and mortality in the developing world [1]. The obligate intracellular Leishmania donovani amastigotes replicate within membrane-bound MP subcellular organelles. Current medical management is not effective for eliciting microbial clearance due to drug resistance, toxicity, bioavailability and cost [2]. Chemotherapy for VL is based on the use of antimony. However, the emergence of resistance has transformed medical management to the use of amphotericin B (AmB) for VL treatment $[2,3]$. Notably, targeted intracellular delivery of $\mathrm{AmB}$ has now emerged as a first-line medical strategy to facilitate pathogen clearance. The develop- ment of improved drug delivery formulations that include nanotechnology-based targeted therapeutics that target infected cells and tissues could improve treatment outcomes [3,4].

It is well accepted that mannose-based carriers can be harnessed to improve antileishmanial drug delivery. This idea is based on the target macrophage surface receptor distribution [2-4]. Proof of concept for such an approach includes, but is not limited to, drugs such as muramyl dipeptide, efavirenz and rifampin [5-7]. Each has yielded encouraging results. Mannose receptors recognize corresponding sugars and facilitate cellular uptake of drug-encased particles [8,9]. Consequent internalization of the therapeutic carrier facilitates drug accumulation at sites of active parasitic infection [8]. Such receptormediated MP drug nanoparticle targeting is linked to the effectiveness of the ligandanchored therapeutic carrier. This serves to improve drug cell entry, retention and cargo
Gul Shahnaz',2, Benson J Edagwa*,2, JoEllyn McMillan'2, Sohail Akhtar ${ }^{3}$, Abida Raza4, Naveeda A Qureshi', Masoom Yasinzai ${ }^{6}$ \& Howard E Gendelman ${ }^{2}$

'Department of Pharmacy, Quaid-i-Azam University Islamabad, 45320, Pakistan ${ }^{2}$ Department of Pharmacology \& Experimental Neuroscience, University of Nebraska Medical Center, Omaha, NE 68198, USA

${ }^{3}$ Department of Entomology, University College of Agriculture \& Environmental Sciences, The Islamia University, Bahawalpur, Pakistan

${ }^{4}$ Nuclear Medicine, Oncology \& Radiotherapy Institute, Islamabad, Pakistan

${ }^{5}$ Department of Animal Sciences, Quaid-i-Azam University, Islamabad, 45320, Pakistan

${ }^{6}$ Centre for Interdisciplinary Research in Basic Sciences, International Islamic University, Islamabad, Pakistan *Author for correspondence: benson.edagwa@unmc.edu 
release in an infected or bystander cell [9]. However, the question of finding the optimal vehicle for drug-based macrophage carriages has not yet been addressed. One answer is chitosan-based nanocarriers. These carriers have gained considerable interest due to their tunable functional groups, biocompatibility and biodegradability [10]. Thiolated chitosan (TC) such as chitosan-thioglycolic acid conjugates can be generated by immobilization of thiol groups on the chitosan polymer backbone [11]. Formation of inter- and intramolecular disulfide bonds within the thiolated polymer matrix results in improved properties, such as in situ gelling/cohesion that facilitates the drug delivery system stability $[12,13]$. Specifically, mannosylation of TC polymer (MTC) is believed to be an appealing strategy for targeted intracellular MP delivery of $\mathrm{AmB}$ as it would not only aid in parasite clearance but also help reduce the dosing volume and frequency. To date, there is only limited research that has been conducted to investigate the potential of mannose-anchored thiolated nanocarriers for VL and linked parasitic disease therapy $[2,3,14]$. In this study, improved mannoseanchored TC (MTC) nanocarriers were developed to facilitate stability, biocompatibility, controlled release and cell uptake by macrophages for antileishmanial therapy.

\section{Materials \& methods}

\section{Materials}

Low MW chitosan, cysteine, hydrogen peroxide, Ellman's reagent (5,5-dithiobis[2-nitrobenzoic acid]), $\mathrm{AmB}$, acetonitrile, methanol, mannose and DMSO were purchased from Sigma-Aldrich (MO, USA). Pooled human serum was obtained from Innovative Biologics (VA, USA). Solvents used were HPLC-grade materials.

\section{TC polymer synthesis}

The synthesis of the thiolated polymer was made through covalent linkage of thioglycolic acid (TGA) to chitosan by amide bond formation between the polymer amino groups and carboxylate groups of the sulfhydryl moiety [12]. Briefly, chitosan $1 \%(\mathrm{w} / \mathrm{v})$ was hydrated with $1 \%(\mathrm{v} / \mathrm{v})$ acetic acid solution. This was by followed the addition of $500 \mathrm{mg}$ of TGA after which 1-ethyl-3-(3-dimethylaminopropyl) carbodiimide hydrochloride-coupling reagent at a final concentration of $100 \mathrm{mM}$ was added to activate the carboxylic acid moieties of TGA. The $\mathrm{pH}$ of the reaction mixture was adjusted to 5.5 with $10 \mathrm{M} \mathrm{NaOH}$, and mixtures were incubated for $3.5 \mathrm{~h}$ under continuous stirring. The solution was dialyzed five-times in a membrane tubing (MW cut-off $12-14 \mathrm{kDa}$ ) for 3 days under protection from light at $10^{\circ} \mathrm{C}$ in order to separate unbound sulf- hydryl moieties from the thiolated polymer. Briefly, the crude thiolated polymer was dialyzed once against 5 $\mathrm{mM} \mathrm{HCl} \mathrm{(5} \mathrm{l})$, then twice against the same media of $1 \%(\mathrm{w} / \mathrm{v}) \mathrm{NaCl}$ to break ionic interactions between the positively charged polymer and the negatively charged sulfhydryl moieties. Finally, the samples were dialyzed thoroughly twice against $1 \mathrm{mM} \mathrm{HCl}$ to adjust the $\mathrm{pH}$ of the TC solution. The dialyzed product was lyophilized and stored at $4^{\circ} \mathrm{C}$ for subsequent use.

\section{MTC synthesis \& characterization}

Mannose was linked onto TC as described with modifications [15]. Mannose immobilization was carried out by reductive amination of TC. Two percent TC was dissolved in $1 \% \mathrm{v} / \mathrm{v}$ aqueous acetic acid by stirring at room temperature. Sodium $0.12 \mathrm{M}$ cyanoborohydride and $0.33 \mathrm{M} \mathrm{D}$-mannose were then added to the resulting viscous polymer solution with vigorous stirring. The resulting reaction mixture was continuously stirred until a white foamy gel was formed. The gel was smashed up then rinsed four-times with $150 \mathrm{ml}$ methanol and once with diethyl ether $(150 \mathrm{ml})$. The light pink solid product was then dried for several hours.

Quantitation of primary amine functional groups at each level of modification was determined by a colorimetric 2,4,6-trinitrobenzenesulfonic acid (TNBS) assay. In this assay, the polymer $(0.5 \mathrm{mg})$ was dissolved in $500 \mu \mathrm{l} \mathrm{NaCl}(0.5 \%$, w/v) solution. After incubation at $25^{\circ} \mathrm{C}$ for $30 \mathrm{~min}, 500 \mu \mathrm{l}$ of TNBS $(0.1 \%$, w/v) comprising $\mathrm{NaHCO}_{3}(4 \%$, w/v) was incorporated into each hydrated aliquot. After $3 \mathrm{~h}$ of incubation at $37^{\circ} \mathrm{C}$ and centrifugation $\left(33,527 \times g ; 5 \mathrm{~min} ; 4^{\circ} \mathrm{C}\right)$, absorbance was recorded at $410 \mathrm{~nm}$ on a microtiter plate reader (Molecular Devices, CA, USA). L-cysteine $\mathrm{HCl}$ standards were used for the calculations.

The degree of conjugation as assessed by the amount of thiol groups anchored to TC and MTC was determined by Ellman's reagent. First, conjugates $(0.5 \mathrm{mg})$ were hydrated in $500 \mu \mathrm{l}$ of phosphate buffer $(0.5 \mathrm{M}$; $\mathrm{pH}$ 8.0). Then $500 \mu \mathrm{l}$ of Ellman's reagent (3 mg in $10 \mathrm{ml}$ of $0.5 \mathrm{M}$ phosphate buffer) was added into each aliquot. The mixtures were incubated for $3 \mathrm{~h}$ at room temperature. The thiol content on TC and MTC was then determined by measuring the absorbance of $300 \mu \mathrm{l}$ of the mixture at $410 \mathrm{~nm}$ on a microtiter plate reader (Molecular Devices). L-cysteine $\mathrm{HCl}$ was used as a standard to quantify the sulfhydryl on the MTC.

The extent of disulfide bond formation due to air oxidation during polymer modifications was measured as previously reported [12]. TC and MTC $(0.5 \mathrm{mg})$ were swelled in Falcon tubes $(15 \mathrm{ml})$ containing $1 \mathrm{ml}$ of 0.05 $\mathrm{M}$ Tris buffer, $\mathrm{pH}$ 6.8. After $30 \mathrm{~min}$ of incubation at room temperature, $1 \mathrm{ml}$ of a $4 \%(\mathrm{w} / \mathrm{v})$ sodium-borohydride solution was added to the reaction mixtures. The 
Table 1. Composition (polymer, surfactant, drug and cross-linking agent ratio) and physicochemical characterization including polydispersity index, particle size, payload and $\zeta$-potential of the ionically cross-linked nanocarriers used in study (designated as 1, 2 and 3 ).

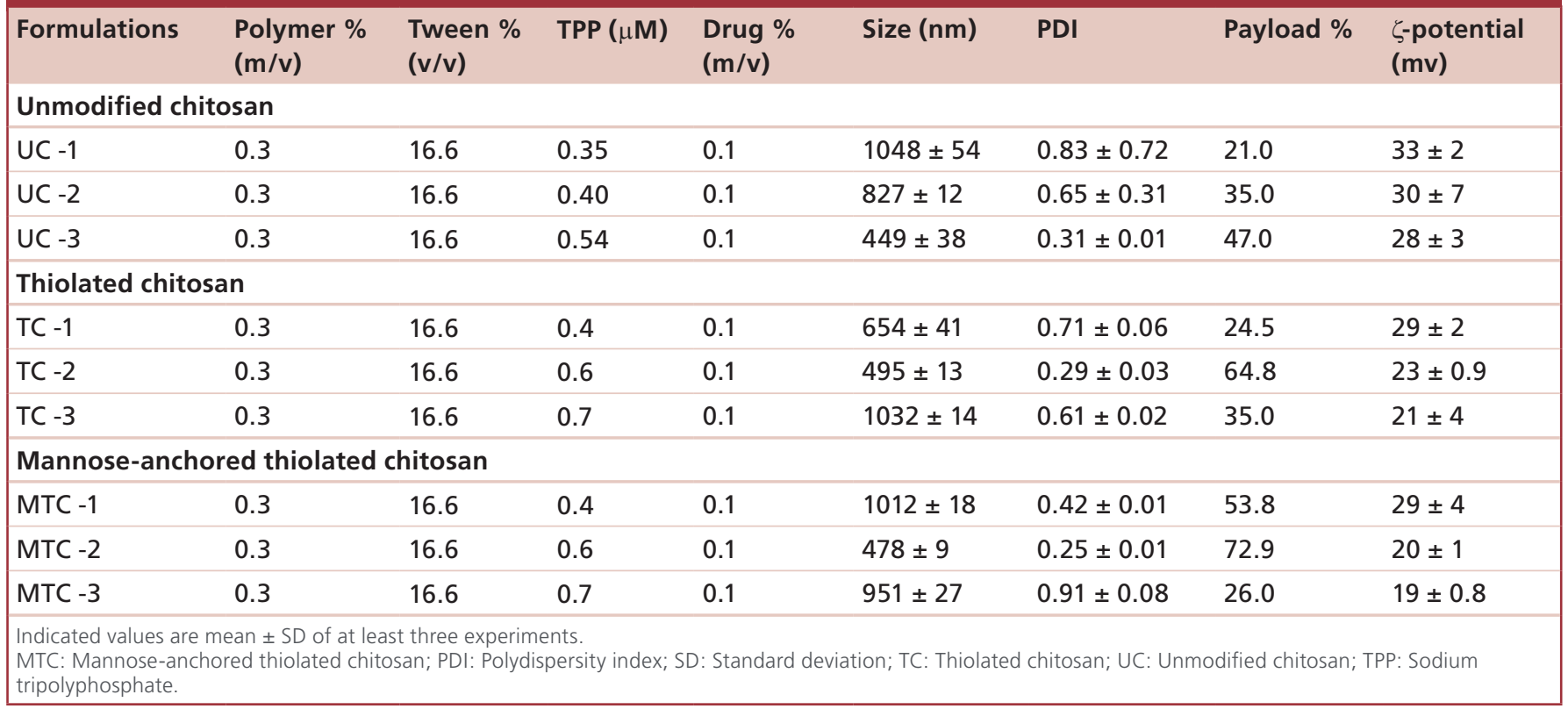

samples were then oscillated for $3 \mathrm{~h}$ in a shaking water bath at $37^{\circ} \mathrm{C}$. The mixture was quenched by the addition of $200 \mu \mathrm{l}$ of $5 \mathrm{M} \mathrm{HCl}$. Ellman's reagent $(500 \mu \mathrm{l}$ of $0.4 \%[\mathrm{w} / \mathrm{v}]$ ) was added to determine the remaining thiol content as described above.

\section{Fourier transform IR \& proton NMR spectroscopy} Immobilization of mannose on TC was elucidated further by Fourier transform IR (FTIR; Schimadzu, Model 8400, Japan) and proton NMR ( ${ }^{1} \mathrm{H}$ NMR; $500 \mathrm{MHz}$; Varian Medical Systems, Inc., CA, USA) spectroscopy. FTIR spectroscopy was acquired by the $\mathrm{KBr}$ disk method over the range of $4000-400 \mathrm{~cm}^{-1}$. ${ }^{1} \mathrm{H}$ NMR spectroscopy was conducted in deuterated DMSO.

\section{Preparation of the nanocarriers}

Nanocarriers of unmodified chitosan (UC), TC and MTC were synthesized using in situ gellation. Specifically, AmB was dissolved in $2.5 \%$ ( $\mathrm{v} / \mathrm{v}$ ) aqueous acetic acid solution and Tween 80 to a final concentration shown in Table 1 . The polymer $(0.33 \%, \mathrm{w} / \mathrm{v})$ was then added to the mixture and the reaction allowed to proceed for $2 \mathrm{~h}$. Afterward the product was mechanically mixed using high-pressure homogenization. The purified nanocarriers were then stabilized by adding an aqueous solution of sodium tripolyphosphate crosslinker at the final concentrations shown in Table 1. The synthesized particles were categorized as either clear, opalescent or agglomerates based on qualitative visual evaluation. The nanocarriers were then partially oxidized with $0.5 \%(\mathrm{v} / \mathrm{v}) \mathrm{H}_{2} \mathrm{O}_{2}$ solution after incubation for $1 \mathrm{~h}$ under continuous stirring at room temperature. The resultant opalescent product of desired physicochemical parameters was selected for further characterization and investigations as shown in Table 2. To prevent particle aggregation during differential centrifugation $\left(33,527 \times g ; 10 \mathrm{~min} ; 4^{\circ} \mathrm{C}\right), 5 \%(\mathrm{w} / \mathrm{v})$ trehalose was added to the nanoformulation before this step. After centrifugation, the supernatant was removed and the pellet washed twice with water before resuspending in $0.1 \%(\mathrm{v} / \mathrm{v})$ aqueous acetic acid solution $(2 \mathrm{ml})$ and stored at $4^{\circ} \mathrm{C}$ for subsequent use.

Particle size, surface charge and polydispersity index (PDI) of the nanocarriers were determined by dynamic light scattering using a Malvern Zetasizer Nano Series Nano-ZS (Malvern Instruments, Inc., MA, USA). Encapsulation efficiency of AmB in UC, TC and MTC was determined after centrifugation $(14,000 \times g, 60$ $\min , 4^{\circ} \mathrm{C}$ ) of nanoparticle suspensions to remove unentrapped insoluble drug. The pelleted nanocarriers were dissolved in methanol and ultrasonicated for $10 \mathrm{~min}$ to facilitate particle rupture. The drug released into the supernatant after centrifugation $(14,000 \times g, 5 \mathrm{~min}$, $4^{\circ} \mathrm{C}$ ) was quantified by HPLC [16]. Drug encapsulation efficiency was calculated using the formula:

$\mathrm{EE}=$

$\frac{\text { Total amount of drug in formulation - Unentrapped drug }}{\text { Total amount of drug }} \times 100$ 
Table 2. Characterization in terms of thiol groups, disulfide bonds, polydispersity index, particle size, payload and $\zeta$-potential of covalently cross-linked (oxidized) nanocarriers.

\begin{tabular}{|c|c|c|c|c|c|c|}
\hline Formulations & $-\mathrm{SH}[\mu \mathrm{Mol} / \mathrm{g}]$ & -S-S- $[\mu \mathrm{Mol} / \mathrm{g}]$ & Size $(\mathrm{nm})$ & PDI & Payload \% & $\zeta$-potential (mv) \\
\hline UC & - & - & $449 \pm 38^{\dagger *}$ & $0.31 \pm 0.01$ & $47.0^{+* *}$ & $28 \pm 4$ \\
\hline TC & $1283 \pm 96$ & $82 \pm 27$ & $495 \pm 13$ & $0.29 \pm 0.03$ & 64.8 & $23 \pm 0.9$ \\
\hline MTC & $1269 \pm 101$ & $62 \pm 18$ & $478 \pm 9$ & $0.25 \pm 0.01$ & 72.9 & $20 \pm 1$ \\
\hline Mannose-anchored Ox & $781 \pm 95$ & $454 \pm 113$ & $362 \pm 27^{\ddagger \star}$ & $0.22 \pm 0.03$ & $78.3^{\ddagger * * *}$ & $19 \pm 3$ \\
\hline
\end{tabular}

Stability of the designed nanocarriers was evaluated under various storage conditions. For stability testing, $100 \mu \mathrm{l}$ aliquots of the nanocarriers were stored at -20 , 4 and $37^{\circ} \mathrm{C}$ over 4 weeks then analyzed for surface charge, size and PDI. Scanning electron microscopy of the nanocarriers was performed using a Hitachi S4700 Field-Emission Scanning Electron Microscope (Hitachi High Technologies America, Inc., IL, USA).

\section{Nanoparticle drug release}

The release profile of AmB from UC, TC and MTC nanocarriers was evaluated at macrophage endosomal and physiological pH, 5.5 and 7.4, respectively. Either sodium acetate buffer or phosphate-buffered saline (PBS), each containing $1 \%(\mathrm{v} / \mathrm{v})$ Tween 80 , was used as the release medium. The release rate of native $\mathrm{AmB}$ powder was evaluated as a control. An appropriate volume of each nanocarrier suspension was reconstituted in $10 \mathrm{ml}$ of release media to achieve a final concentration of $10 \mathrm{mg} / \mathrm{ml}$ and transferred into dialysis bags (MW cut-off $12-14 \mathrm{kDa}$ ). The dialysis bags were then immersed in $100 \mathrm{ml}$ of release media and stirred at $37^{\circ} \mathrm{C}$. At predetermined time intervals, an aliquot of the medium was drawn and diluted in methanol. The concentration of drug released was determined by HPLC [16].

\section{Parasites, cells \& nanoparticle treatments} The WHO reference strain of $L$. donovani promastigotes (MHOM/IN/80/DD8) was obtained from American Type Culture Collection $\left(\right.$ ATCC $^{\circledR}$ 50212) and is sensitive to $A m B$. The resistance in the acquired strain was provoked by incremental increases in $\mathrm{AmB}$ concentration enabling the pathogen to grow at $500 \mu \mathrm{M}$ drug concentration. Resistant and sensitive $L$. donovani clinical isolate parasites $\left(1-2 \times 10^{6}\right.$ cells $\left./ \mathrm{ml}\right)$ were grown at $25^{\circ} \mathrm{C}$ in Medium 199 with Hank's Balanced Salts (Thermo Fisher Scientific, MA, USA), plus 10\% (v/v) heat-inactivated fetal bovine serum; American Type Culture Collection cat. no. 30-2020) and $50 \mathrm{mg} / \mathrm{l}$ gen- tamicin, in a $75 \mathrm{~cm}^{2}$ tissue culture flask ( $14 \mathrm{ml}$ medium) for $>4$ days to stationery phase $\left(50-100 \times 10^{6}\right.$ cells/ $\mathrm{ml})$. Macrophage J774 cells were grown in RPMI-1640 medium (HyClone; GE Healthcare Life Sciences, UT, USA) supplemented with $15 \%(\mathrm{v} / \mathrm{v})$ heat-inactivated fetal bovine serum and $50 \mathrm{mg} / \mathrm{l}$ gentamicin at $37^{\circ} \mathrm{C}$. The effects of the nanoformulations on cell viability of J774 cells and primary human monocyte-derived macrophages were evaluated using the 3-(4,5-dimethyl2-thiazolyl)-2,5-diphenyl-2H-tetrazoliumbromide (MTT) assay with minor modifications [2]. Briefly, noninfected J774 cells were seeded at a density of $1 \times 10^{6}$ cells $/ \mathrm{ml}$ in 96-well plates at a volume of $200 \mu \mathrm{l}$ culture medium/well and incubated for $24 \mathrm{~h}$ at $37^{\circ} \mathrm{C}$. Depending on surface chemistry nanoformulations had variant encapsulation/associations of $\mathrm{AmB}$ with the polymeric nanocarriers; the drug loading capacities were $80 \%$, $70 \%$, and $47 \%$ for MTC, TC, and Chito, respectively. To maintain equivalent concentrations, nanoformulations of MTC, TC and UC were dissolved in $100 \%$ DMSO and diluted to reach concentrations of 25,50 and $75 \mu \mathrm{g} / \mathrm{ml} \mathrm{AmB}$ in the culture medium. The maximum concentration of DMSO after dilution was $0.1 \%$. Cells treated with 25,50 or $75 \mu \mathrm{g} / \mathrm{ml}$ of native AmB or no drug constituted control groups. The cells were incubated for 24,48 or $72 \mathrm{~h}$ at $37^{\circ} \mathrm{C}$ in a $5 \% \mathrm{CO}_{2}$ incubator after nanocarrier treatment. Following incubation, the supernatant was replaced with culture medium containing MTT $(500 \mu \mathrm{g} / \mathrm{ml})$ and further incubated for $4 \mathrm{~h}$ at $37^{\circ} \mathrm{C}$. To dissolve formazan crystals, $100 \mu \mathrm{l}$ of DMSO was added and absorbance was measured at $450 \mathrm{~nm}$ using a microplate reader.

J774 cell uptake and retention of nanocarriers, AmBisome ${ }^{\circledast}$ and native drug were evaluated as previously described [17]. Briefly, cells were incubated with nanocarriers at $100 \mu \mathrm{M}$ drug concentrations, and cellular uptake measured over a $12 \mathrm{~h}$ period. Adherent cells were washed three-times with PBS and scraped. Cell pellets were collected by centrifugation at $950 \times g$ for $10 \mathrm{~min}$ then resuspended in $200 \mu \mathrm{l}$ of methanol, 
sonicated and centrifuged. Triplicate $20-\mu \mathrm{l}$ samples of methanol extracts were assessed by HPLC [16]. For determination of efflux pump inhibition, J774 cells infected with $L$. donovani were treated with $5 \mu \mathrm{M}$ verapamil, an adenosine triphosphate-binding cassette (ABC) transporter inhibitor. Verapamil was added to the cells with or without parasites and incubated for $2 \mathrm{~h}$ prior to treatment with nanoformulations or AmB. After treatment with verapamil the cells were washed with PBS ( $\mathrm{pH}$ 7.2) and treated with nanoformulations, AmBisome or AmB then processed for microbial uptake [17]. For cell drug retention studies, J774 cells were incubated with nanoformulations for $24 \mathrm{~h}$ and washed three-times with PBS. Fresh medium without nanoformulations was added and the cells were cultured for a further 10 days with half medium changes every other day. On days 2, 4, 8 and 10 following nanoformulation treatment, J774 cells were scraped then processed for drug quantitation by HPLC as described for cell uptake.

\section{Antileishmanial activities}

An amastigote model in a macrophage cell line was used to evaluate antileishmanial activity of the developed formulations and AmBisome, as reported previously [18]. Briefly, the J774 cells were resuspended (2.5 × $10^{5}$ cells $/ \mathrm{ml}$ ) in RPMI-1640 culture medium without serum. The cells were plated onto 8-well Lab-Tek CCR ${ }^{2}$ tissue culture slides (NUNC; Thermo Fisher Scientific) at a density of $200 \times 10^{3}$ cells/well and incubated at $37^{\circ} \mathrm{C}$ for $24 \mathrm{~h}$ in a humidified incubator. The cells were then washed twice with serum-free medium and infected with $100 \mu \mathrm{l}$ metacyclic stage of $L$. donovani at an infection ratio of 10:1 (parasites/macrophages) in $200 \mu \mathrm{l}$ of whole medium (RPMI $1640+10 \%$ heatinactivated fetal calf serum $+50 \mathrm{mg} / \mathrm{l}$ gentamicin) and incubated for $12 \mathrm{~h}$. Nonphagocytosed parasites were removed by washing three-times with PBS and the wells were resupplemented with RPMI-1640 complete medium. Stock solutions of native AmB and UC, TC, MTC nanocarriers were prepared in 100\% DMSO at $1 \mathrm{mg} / \mathrm{ml} \mathrm{AmB}$. AmBisome was reconstituted consistent with the manufacturer's protocol to achieve a $5 \mathrm{mg} / \mathrm{ml}$ stock of AmB. Working concentrations were prepared in whole medium (RPMI $1640+10 \%$ heatinactivated fetal calf serum $+50 \mathrm{mg} / \mathrm{l}$ gentamicin). The cells were treated with AmB formulations (Chito, CT, MCT and AmBisome) at six different drug concentrations $(1-0.004 \mu \mathrm{g} / \mathrm{ml} \mathrm{AmB})$ prepared by serial dilution. Untreated infected macrophages were used as positive controls. The drug activities of free UC, TC and MTC nanocarriers were also investigated. Each formulation concentration was tested in quadruplicate. The maximum DMSO concentration of $0.1 \%$ was found to have no influence on macrophage/amastigote clearance. After $72 \mathrm{~h}$ of incubation $\left(5 \% \mathrm{CO}_{2}\right.$ at $37^{\circ} \mathrm{C}$ ), slides were fixed with $100 \%$ methanol for $1 \mathrm{~min}$ and stained for $10 \mathrm{~min}$ with $10 \%$ Giemsa's solution. Giemsa-stained intramacrophage amastigotes slides were visualized under a light microscope (Zeiss, AXIO, NY, USA). Percent inhibition from test formulations and $\mathrm{AmB}$ were calculated as cells/100 nucleated nontreated control cells. Data were fitted using the nonlinear dose-response sigmoidal curve, and the $\mathrm{IC}_{50}$ values were estimated by using Microsoft xl/fit.

In vivo therapeutic efficacy evaluations of the developed nanoformulations were performed according to a protocol approved by the Institutional Animals Ethical Committee of Quaid-i-Azam University, Islamabad, Pakistan. Thirty-six BALB/c mice $(20-25 \mathrm{~g})$ were infected by intracardiac injection of $1 \times 10^{8}$ promastigotes of $L$. donovani. After 4 weeks, infection was confirmed in three randomly selected mice by Giemsa staining of splenic tissue. Native-free drug AmB (group I) and nanoformulated UC (group II), TC (group III), MTC (group IV) and drug-free nanocarrier (group V) were administered orally at $1 \mathrm{mg} / \mathrm{kg}$ body weight per day for 7 days; whereas the control group (group VI) received an equal volume of saline. In order to validate the data, a single intravenous dose of $\mathrm{AmB}$ was administered at $1 \mathrm{mg} / \mathrm{kg}$ body weight/day for 7 consecutive days. Treated groups were sacrificed on day 7 posttreatment and compared with untreated infected control animals. The spleen weight was calculated after autopsy and tissue smears were prepared for microscopic examination by Giemsa staining. The percent suppression of splenic parasite load and parasite burden and the percent inhibition of parasite replication were determined using the following formula:

$\mathrm{PI}=\frac{\mathrm{PP}-\mathrm{PT}}{\mathrm{PP}} \times 100$

where PI is the percent inhibition, PP is the amount of amastigotes/100 macrophage nuclei pretreatment while $\mathrm{PT}$ is the amount of amastigotes $/ 100$ macrophage nuclei post-treatment in spleen tissue smears.

\section{Statistical analyses}

Data sets were investigated for normal distribution by using the Kolmogorov-Smirnov test. Normally distributed data were analyzed using one-way analysis of variance followed by the Tukey's posthoc test. Kruskal-Wallis tests were followed by Dunn's post hoc evaluations for non-normally distributed data. All results were expressed as mean \pm standard deviation $(\mathrm{SD})$, with $\mathrm{n}$ representing the number of repeats. Twotailed statistical analyses were considered significant at $\mathrm{p}<0.05$ or highly significant at $\mathrm{p}<0.01$. 


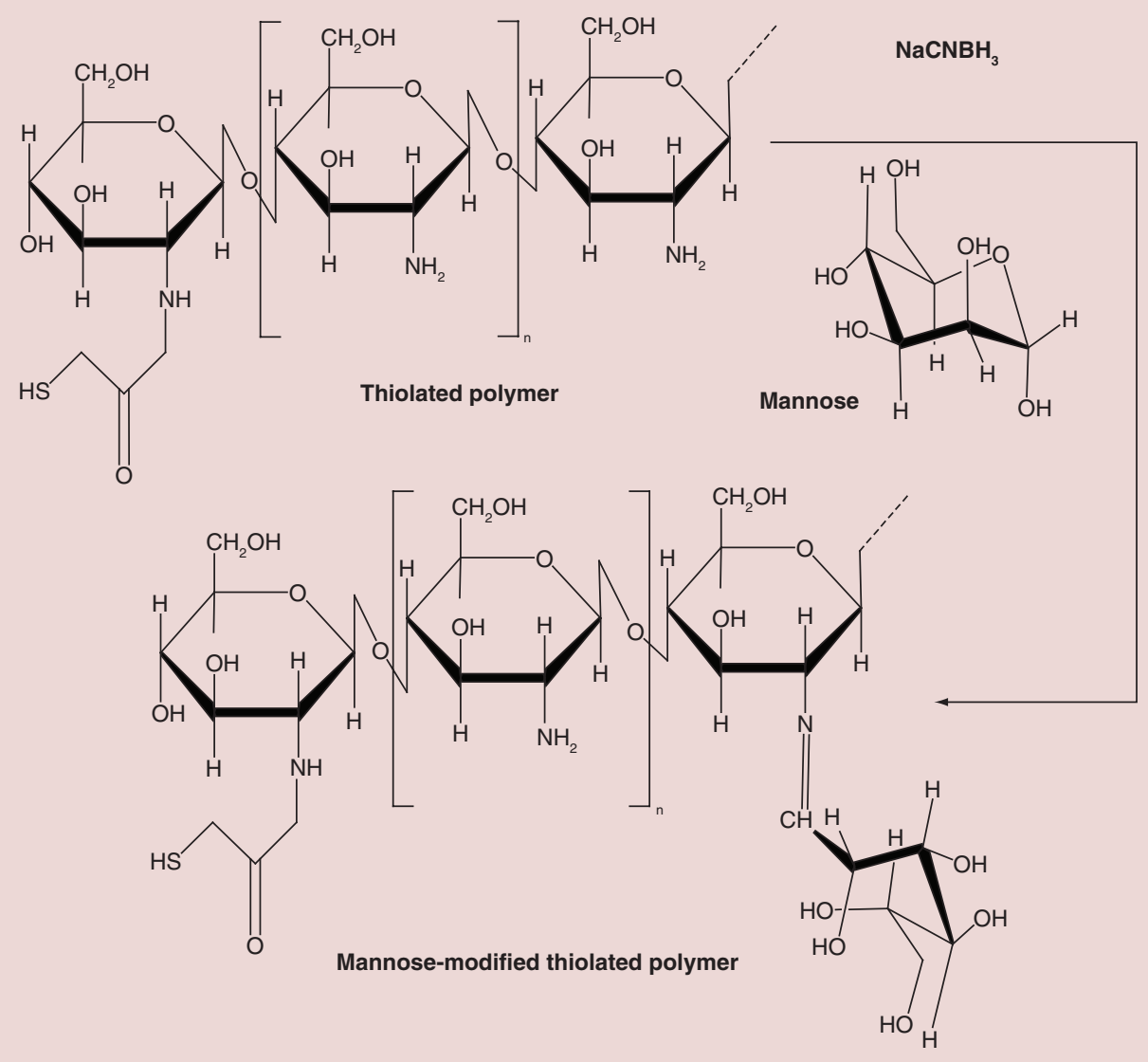

Figure 1. Synthetic scheme for the establishment of mannose-modified chitosan-thioglycolic acid conjugate. The Schiff's base $(\mathrm{R}-\mathrm{CH}=\mathrm{N}-\mathrm{R})$ mediated amide bonds formation between the aldehyde group of mannose and the amino group of the thiolated polymer.

\section{Results}

\section{TC synthesis \& characterization}

Evaluation of the thiol groups immobilized to the thiolated polymer by the Ellman's test demonstrated that on average $1301 \pm 194 \mu \mathrm{mol}$ of thiol groups were attached/gram of polymer (mean $\pm S D ; n=3$ ). In addition, the amounts of primary amino groups and disulfide bonds within the TC were estimated by TNBS and disulfide bond assays, respectively. According to the data, there were $429 \pm 62 \mu \mathrm{mol}$ primary amino groups per gram of polymer (mean $\pm S D ; n=3$ ) and $107 \pm 12 \mu \mathrm{mol}$ disulfide bonds/gram of polymer (mean $\pm \mathrm{SD} ; \mathrm{n}=3$ ). The reduced disulfide bond formation implied reduced oxidation that had occurred during the conjugation reaction. This demonstrated the high efficiency of the thiolation process. The efficiency of the purification step for the TC polymer was con- firmed by comparison to control polymers synthesized similarly but without 1-ethyl-3-(3-dimethylaminopropyl) carbodiimide hydrochloride during the conjugation step. The control polymers exhibited negligible quantities of thiol groups per gram of polymer. The purified polymers were frozen and lyophilized to give white fibrous structures that were readily soluble in aqueous solutions.

\section{MTC nanocarriers}

We next sought to determine the utility of thiolated polymer for MP targeting as a therapeutic strategy as a therapy for VL. Mannose modification of the TC polymer was initiated by ring opening of mannose followed by reductive amination reaction of the resultant aldehyde with free amino groups on the TC polymer. The synthetic scheme for this mannose-modified chi-

Figure 2. Characterization of the polymers by nuclear magnetic resonance spectroscopy (see facing page). 1H-NMR spectra comparing unmodified polymer (A) and thiolated chitosan (B) polymer controls to mannose-anchored thiolated chitosan (C). The presence of methylene peaks (3.5-3.9 ppm and 4.5-5.1 ppm) in the spectrum of the mannose-anchored thiolated chitosan confirmed the attachment of the sugar to the polymer. 


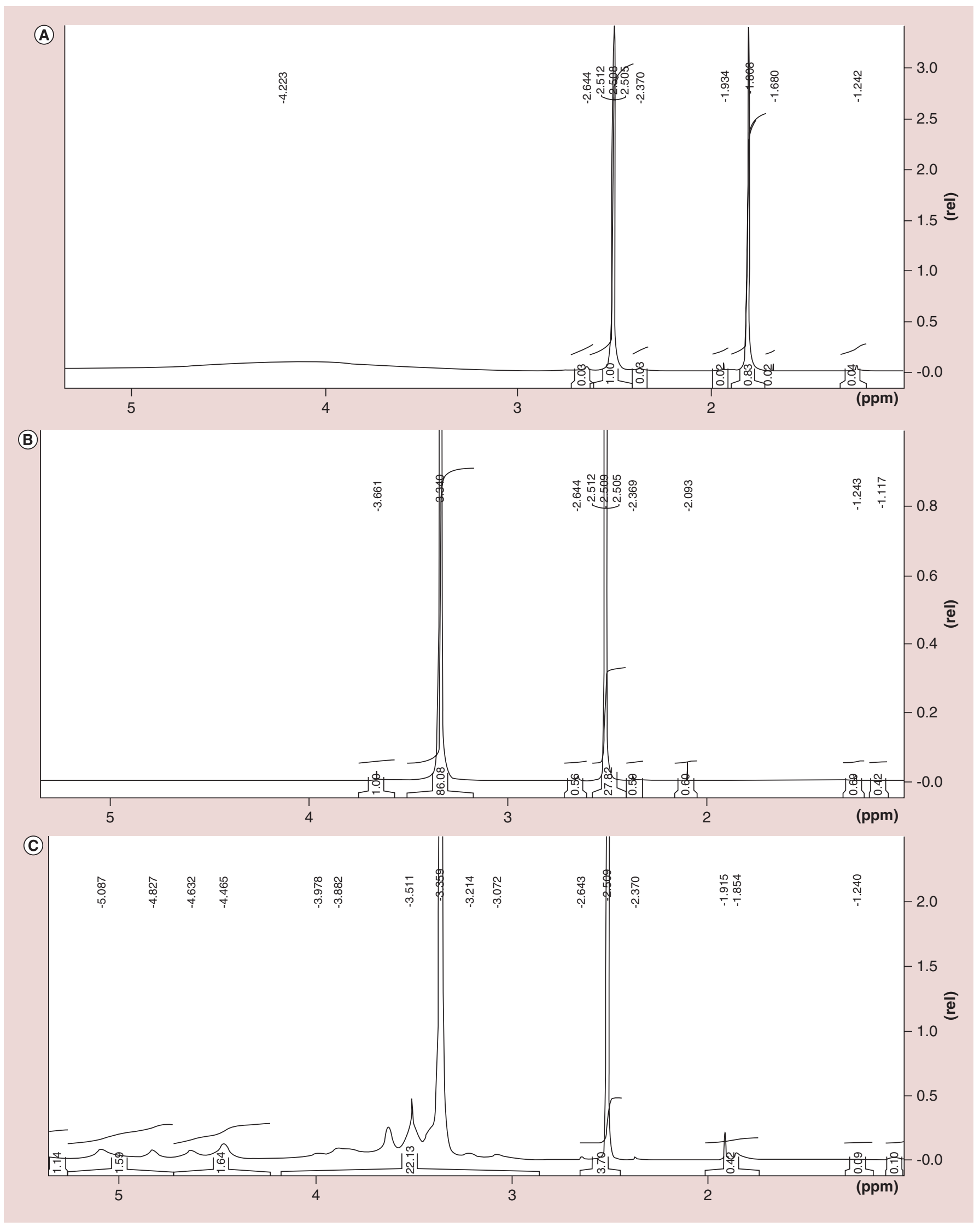




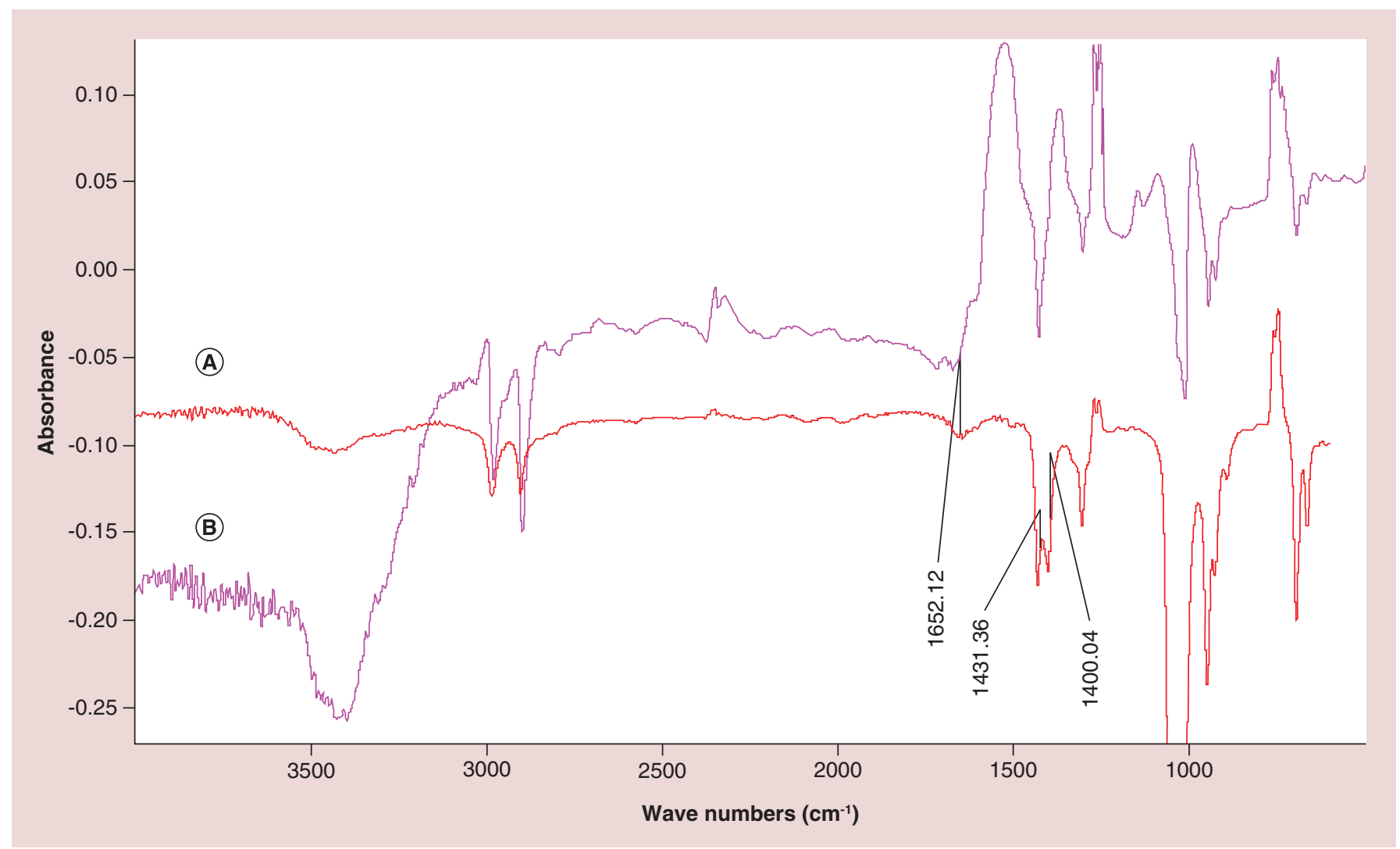

Figure 3. Characterization of the polymers by fourier transform infrared spectroscopy. Fourier transform IR spectra of thiolated chitosan (A) and mannose-modified thiolated chitosan (B). The presence of amide carbonyl and hydroxyl bands in FTIR spectrum of the mannose-anchored thiolated polymer further confirms the association of the mannose sugar and the polymer backbone.

tion was induced by the addition of an aqueous solution of $\mathrm{H}_{2} \mathrm{O}_{2}$. Consequently, a decrease in the density of thiol groups within the nanocarriers was confirmed using the Ellman's reagent. Stability of the developed nanoformulations in terms of size, charge and PDI was evaluated under various storage temperatures $(-20,4$ and $37^{\circ} \mathrm{C}$ ) in a time-dependent manner. No notable changes in size, $\zeta$-potential and PDI, were observed when the nanoformulations were kept for a period of 4 weeks at 4 and $37^{\circ} \mathrm{C}$. However, statistically significant increases in average size and PDI ( 2- and 1.5fold, respectively) were observed for nanoformulations after 4 weeks at $-20^{\circ} \mathrm{C}$.

\section{Drug release profiles}

In this study, $\mathrm{AmB}$ release profiles of native drug powder, UC, TC and MTC nanocarriers were evaluated for 10 days at endosomal $\mathrm{pH}$ of 5.5 and physiological $\mathrm{pH}$ of 7.4. When assessing in vitro drug release of a hydrophobic drug, sink conditions were maintained. These conditions were maintained by inclusion of $1 \%(\mathrm{v} / \mathrm{v})$ Tween in the phosphate buffer [17,19]. This allowed the total quantity of drug can be eluted from the nanocarriers and to reduce the adhesion of $\mathrm{AmB}$ onto the dialyzing membrane wall. The percentage of $\mathrm{AmB}$ released from the nanocarriers was evaluated in a time-dependent manner (Figure 5). The release patterns were biphasic (i.e., an initial burst release of $40 \%$ within 2 days followed by an extended sustained release for 10 days). Moreover, as observed from native drug powderm $100 \%$ drug was released in both settings within $1 \mathrm{~h}$ (data not shown). Therefore, release media provides a sufficient hydrophobic environment for complete dissolution of drug. The initial burst release of the drug might be the result of diffusion of loosely bound drug near the particle surface. During the second phase, both TC and MTC nanocarriers exhibited a slow sustained release of $\mathrm{AmB}$ for up to 10 days, compared with $>80 \%$ of drug released from UC nanocarriers within 4 days, possibly due to poor stability of the UC polymeric network. It is also worth noting that both TC and MTC nanocarriers exhibited the lowest initial drug release as compared with UC.

\section{Biocompatibility screening studies}

The biocompatibility of the developed nanoformulation was evaluated in J774 cells using the MTT assay. 
tosan-TGA conjugate is illustrated in Figure 1. Successful synthesis of the conjugate was confirmed by ${ }^{1} \mathrm{H}$ NMR and FTIR spectroscopy. The ${ }^{1} \mathrm{H}$ NMR spectrum of MCT is shown in Figure 2. Peaks in the region of 3.5-3.9 ppm and 4.5-5.1 ppm correspond to methylene protons $\left(\mathrm{CH}_{2}\right.$ group) of the mannose sugar. These were present in the final product spectrum but not in the starting material (Figure 2A \& B, respectively). The FTIR spectra of the TC and mannose-modified TC are illustrated in Figure 3. A characteristic band at $3363 \mathrm{~cm}^{-1}$ corresponds to stretching vibrations of $-\mathrm{NH}_{2}$ and $-\mathrm{OH}$ functional groups. The band at $2375 \mathrm{~cm}^{-1}$ corresponding to thiol groups was observed in the TC spectrum (Figure 3). For the mannose-modified TC, new peaks at 1652, $1431 \mathrm{~cm}^{-1}$ and $1400 \mathrm{~cm}^{-1}$ corresponds to amide bond formation and $-\mathrm{OH}$ stretching vibrations, an indication that the mannose sugar was incorporated on the polymer backbone.
AmB loaded UC, TC and MTC nanocarriers were manufactured by a blend of embedding and diffusion. The payload of AmB-loaded nanocarriers was from 21 to $72 \%$ (Table 1). Based on our prior experience, $0.54 \mu \mathrm{M} \mathrm{TPP}$ is required for cross-linking Chito and $0.60 \mu \mathrm{M}$ for TC and MTC nanocarriers to stabilize the particles. The morphologies of the synthesized nanocarriers were rod-shaped (Figure 4). Nanoparticle surface chemistry and parameters influence nanoformulation stability and attachment onto biological surfaces. The average $\zeta$-potentials of MTC $(19 \pm 3)$ and TC $(21 \pm 0.6)$ were found to be lower than that of UC $(28 \pm 4)(p<0.05)$ nanocarriers. The decrease in the $\zeta$-potential of MTC was the result of mannose immobilization onto the polymer backbone. Moreover, all the nanoparticles were found to have a moderate PDI and narrow size distribution as illustrated in Table 2. To further stabilize TC and MTC nanocarriers, inter- and intramolecular disulfide bond forma-

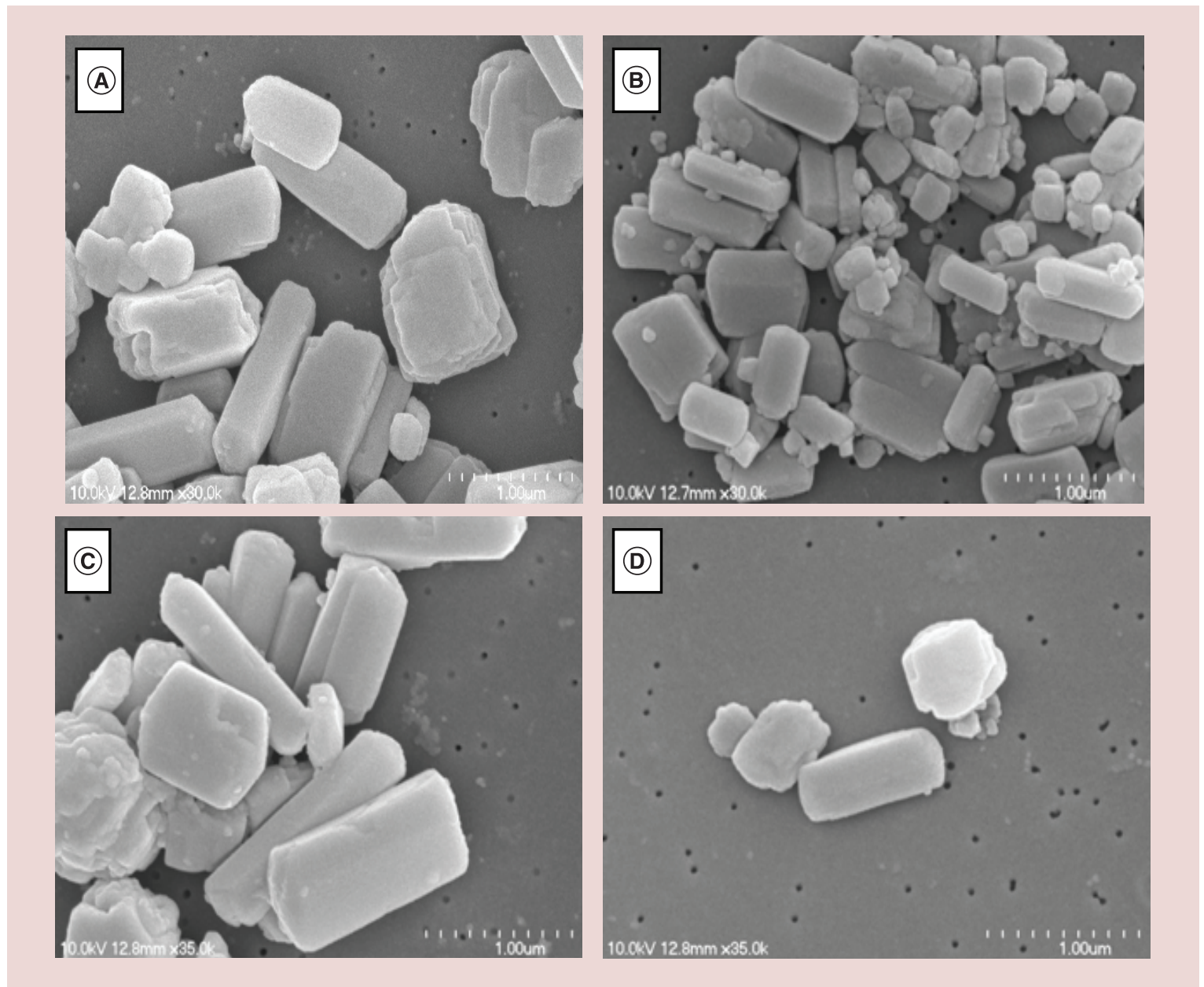

Figure 4. Nanoparticle morphologies. SEM images of unmodified chitosan (A), thiolated chitosan (B) and mannose-anchored thiolated chitosan (C) nanocarriers before centrifugation and SEM image of mannoseanchored thiolated chitosan (D) after centrifugation.

SEM: Scanning electron microscopy. 


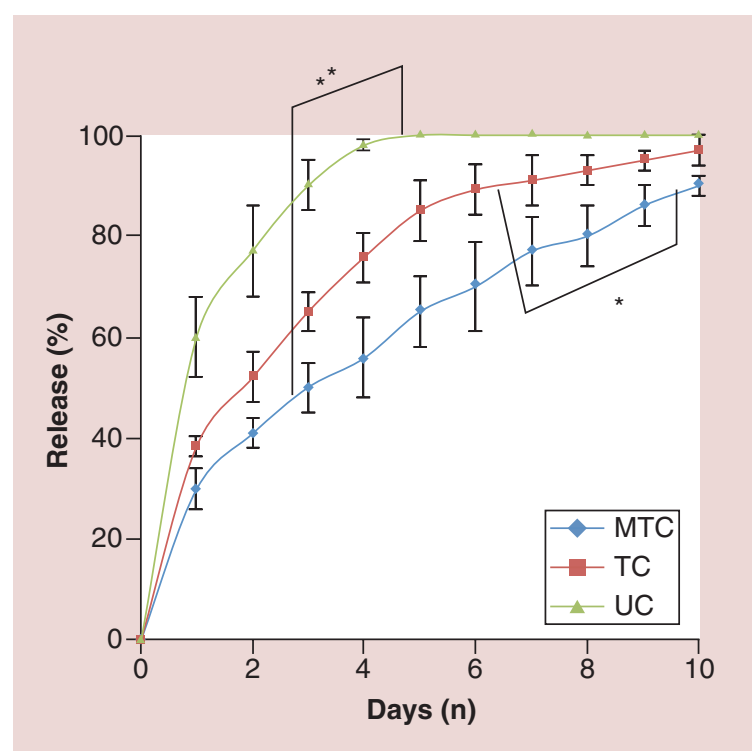

Figure 5. Release profile of developed

nanoformulations. Dissolution studies were performed in phosphate buffer pH 7.2 at $37^{\circ} \mathrm{C}$. Values are mean \pm SD of three experiments. Statistically significant differences in the release profile of MTC were determined compared with TC and UC at significance levels of ${ }^{*} p<0.05 ; * * p 0.01$.

MTC: Mannose-anchored thiolated chitosan; SD: Standard deviation; TC: Thiolated chitosan; UC: Unmodified chitosan.

J774 cells were incubated with various concentrations of $\mathrm{AmB}$ nanoformulations $(0,25,50$ and $75 \mu \mathrm{g} / \mathrm{ml}$ $\mathrm{AmB})$. Differences were observed in the cytotoxicity profiles between TC and MTC, UC $(\mathrm{p} \leq 0.05)$ and native drug $(\mathrm{p} \leq 0.05)$. With MTC $>90 \pm 4.3 \%$ cell survival over $72 \mathrm{~h}$ was observed at the highest concentration tested $(75 \mu \mathrm{g} / \mathrm{ml} \mathrm{AmB})$, while native drug solution reduced cell viability to $34.5 \pm 5.6 \%(\mathrm{n}=3 \pm$ $\mathrm{SD}$ ) as shown in Figure 6. The values for the negative control Triton X-100 (2\% v/v) and the positive-control medium without fetal calf serum/phenol red showed $0.40 \pm 0.03 \%$ and $100.8 \pm 4 \%$ viability, respectively.

\section{Internalization of MTC AmB nanocarriers by macrophages}

Cellular internalization of targeted (TC and MTC), nontargeted (UC), AmBisome and native drug after $12 \mathrm{~h}$ incubation was compared in sensitive and resistant $L$. donovani infected or uninfected control cells following drug nanoformulation treatments $(100 \mu \mathrm{M})$. UC nanocarriers, AmBisome and native drug showed reduced uptake into infected cells with the resistant $\mathrm{VL}$ strain after 12-h incubation $(\mathrm{p} \leq 0.05)$ (Table 3). To determine whether MDR1 is involved in the efflux of $A \mathrm{mB}$, pretreatment with verapamil, an inhibitor of MDR1 was tested. Here retention of AmB from UC, AmBisome and native drug solution in infected and uninfected macrophages was evaluated (Table 3). Pretreatment with verapamil demonstrated partial reversal of the efflux process, as the uptake of $\mathrm{AmB}$ was 2-, 2.5-, 1.2-fold higher from UC, AmBisome and native drug solution, respectively, for the resistant strain-infected macrophages. However, the uptake of the uninfected macrophages and sensitive strain-infected macrophages was not altered significantly by pretreatment with verapamil. These observations suggest that the lower intracellular AmB concentration in the resistant straininfected macrophages compared with noninfected macrophages and sensitive strain-infected macrophages may be the result of upregulated drug efflux machinery. TC and MTC nanocarriers $(100 \mu \mathrm{M})$ were rapidly internalized into the noninfected macrophages and drug concentrations of up to $12.9 \pm 2.3 \mu \mathrm{g}$ of $\mathrm{AmB} / 10^{6}$ cells and $28.6 \pm 1.4 \mu \mathrm{g} \mathrm{AmB} / 10^{6}$ cells over $12 \mathrm{~h}$, respectively. We observed the uptake of TC and MTC to be 4.2- and 9.6-fold higher, respectively, in resistant strain-infected macrophages compared with unmodified nonthiolated nanocarriers (Table 3). The results reported in this work therefore suggest that the thiolated surface-modified nanocarriers contribute to inhibition of $\mathrm{AmB}$ efflux in resistant strain-infected macrophages. MTC nanoparticles showed 71.5-, 26.0-, 4.4- and 2.2-fold increases in uptake by noninfected macrophages at $12 \mathrm{~h}$ compared with equimolar concentrations of TC $(\mathrm{p} \leq 0.05)$, $\mathrm{UC}(\mathrm{p} \leq 0.05)$, AmBisome $(\mathrm{p} \leq 0.05)$ and native drug $(\mathrm{p} \leq 0.001)$, respectively, Table 3.

The amount of drug lost during the washing steps of cellular internalization was determined by HPLC analysis of the supernatants. It was observed that loss of drug in the washed volume was decreased with longer incubation time (data not shown). Interactions of the nanocarriers with the cell surface seem to be time dependent. Moreover, UC, AmBisome and native drug displayed higher drug loss in contrast to TC and MTC nanocarriers.

We also investigated the drug retention profile of the developed nanocarriers over 10 days (Figure 7). There were significant differences in the drug retention profile ( $p \leq 0.05)$ between AmBisome and native drug when cells were treated with equimolar drug concentrations. Specifically, $0.34 \mu \mathrm{g}$ of $\mathrm{AmB} / 10^{6}$ cells was detected 2 days after treatment with AmBisome, compared with none for the native drug. UC nanocarriers were found to keep the drug inside the cells for 8 days. The TC and MTC nanocarriers retained and slowly released AmB over the 10-day period.

\section{Antileishmanial activities}

In this study, various AmB nanoformulations were synthesized and investigated against $L$. donovani amastigotes in a concentration-dependent manner (Figure 8). 
Free $\mathrm{AmB}$ and $\mathrm{AmBisome}$ were used as controls. Drugfree nanocarriers (UC, TC and MTC) were used as additional controls. As shown in Figure $8 \mathrm{~A}, \mathrm{AmB}$, AmBisome UC, TC and MTC nanocarriers at doses equivalent to $1.0 \mu \mathrm{g} / \mathrm{ml} \mathrm{AmB}$ showed $48 \% \pm 6 \%, 60 \%$ $\pm 11 \%, 76 \% \pm 9 \%, 84 \% \pm 7 \%$ and $96 \% \pm 2 \%$ inhibition of parasites, respectively. The measured $\mathrm{IC}_{50}$ of AmB, AmBisome, UC, TC and MTC nanocarriers was found to be $0.256 \pm 0.013 \mu \mathrm{g} / \mathrm{ml}, 0.208 \pm 0.01$ $\mu \mathrm{g} / \mathrm{ml}, 0.164 \pm 0.03 \mu \mathrm{g} / \mathrm{ml}, 0.096 \pm 0.004 \mu \mathrm{g} / \mathrm{ml}$ and $0.019 \pm 0.007 \mu \mathrm{g} / \mathrm{ml}$, respectively. Macrophage targeting through mannose-anchored thiolated nanocarriers significantly improved the antileishmanial activity of AmB against intracellular parasites. Further evaluation of the potential application and future development of the synthesized nanoformulations as slow acting antileshmania therapies were conducted over a 10-day period. The native drug exhibited limited antimicrobial responses (data not shown). In contrast, MTC showed extended activity against $L$. donovani, suppressing parasite replication by 3.4 -fold on the 10th day.

The in vivo therapeutic efficacy of MTC nanocarriers at a single dose of $1 \mathrm{mg} \mathrm{AmB} / \mathrm{kg}$ was evaluated in $L$. donovani-infected mice and compared with native $A m B$. The observed findings were then validated with AmBisome. The results showed that MTC nanocarriers were considerably more effective $(89 \% \pm 7 \%$ inhibition) than $\mathrm{AmB}(17 \% \pm 4 \%$ inhibition) $(\mathrm{p}<0.001)$. Whereas TC, UC, AmBisome and nanocarriers alone had $63 \% \pm 5 \%, 36 \% \pm 2 \%, 19 \% \pm 2.4 \%$ and $11 \%$ $\pm 1 \%$ reductions in parasites, respectively. Among all tested formulations, MTC demonstrated the greatest antileishmanial efficacy.

\section{Discussion}

For a spectrum of infectious diseases that include leishmaniasis, tuberculosis and HIV, macrophages serve as critical reservoirs and site of microbial replication for the intracellular microorganisms. Limited access of therapeutic agents to intracellular sites remains a major obstacle to effective anti-infectious disease therapy. In addition to reducing nonspecific drug toxicity, selective targeting of intramacrophage parasites with ligandanchored nanocarrier delivery systems would facilitate pathogen clearance by ensuring that drug concentrations at these sites remain within the desired therapeutic range. In order to achieve this goal, mannose receptor-mediated endocytosis of drug nanocarriers by macrophages has become increasingly attractive. In the present study, MTC nanocarriers were synthesized, characterized and evaluated against intramacrophage amastigote parasites.

Chitosan-based nanocarriers are widely used for drug delivery applications owing to their efficient intracellular release of drug payloads and biocompatibility. Thiolated polymers such as TC [20] promote nanoparticle internalization owing to their unique properties. Mannose modification of the TC polymer was used to improve uptake of the drug carrying particles by macrophages where Leishmania amastigotes reside. Nanocarriers for macrophage delivery may offer numerous advantages over traditional delivery, including enhanced treatment rates, reduced off-target adverse effects, amplified drug stability and successful intracellular targeting. The MTC nanocarriers were manufactured and characterized by various analytical techniques, including FTIR, ${ }^{1} \mathrm{H}$ NMR and scanning electron microscopy. The results of mannose anchoring were consistent with the earlier described mannosylation of nanocarriers with amino groups of polymer [2]. UC, TC and MTC nanocarriers were formulated by homogenization followed by ionic gelation with TPP and generation of intra- and intermolecular disulfide bonds. Rod-shaped morphologies of the nanocarriers could be due to a par-

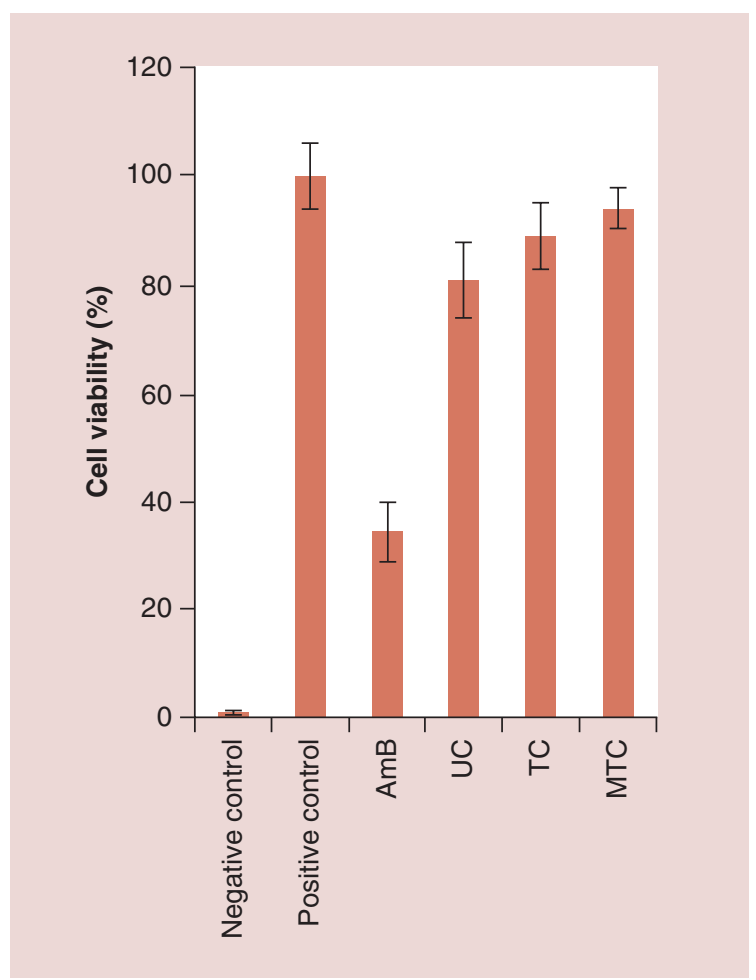

Figure 6. Biocompatibility of nanoformulations in J744A.1 cells after $72 \mathrm{~h}$ incubation. To retain equivalent concentrations, nanoformulations of MTC $(0.93 \mu \mathrm{g} / \mathrm{ml})$, TC $(1 \mu \mathrm{g} / \mathrm{ml})$ and UC $(1.6 \mu \mathrm{g} / \mathrm{ml})$ were dissolved to achieve concentrations of $75 \mu \mathrm{g} / \mathrm{ml} \mathrm{AmB}$ in the culture medium. Results are expressed as mean \pm SD of three replicate wells at significance levels of $* * p<0.01 ; * * * p<0.001$. MTC: Mannose-anchored thiolated chitosan;

SD: Standard deviation; TC: Thiolated chitosan; UC: Unmodified chitosan. 
ticular characteristic of homogenized hydrophobic drug particles.

For in vivo applications, it is highly desirable to design a carrier that is stable and provides sustained release of therapeutic agents during systemic circulation. In the systemic circulation, the stability of the drug carrier is key to minimizing rapid elimination of the drug from the body. Hence, drug release was carried out at endosomal $\mathrm{pH}$ of 5.5 and physiological $\mathrm{pH}$ of 7.4 containing $1 \%(\mathrm{v} / \mathrm{v})$ Tween- 80 . An initial burst release of drug ( $\sim 40 \%$ within 2 days) was observed with TC and MTC nanocarriers followed by a slowrelease phase, which continued for 10 days. In contrast, UC nanocarriers released $80 \%$ of drug within 4 days. A possible explanation for the observed sustained drug release from TC and MTC nanocarriers may be due to covalent cross-linking of disulfide bonds formed within the modified polymer matrix during the swelling process [21]. Moreover, all developed nanocarriers released $>55 \%$ of drug at endosomal pH 5.5 (data not shown). This faster release at endosomal $\mathrm{pH}$ may be the result of increased solubility of $\mathrm{AmB}$ and the polymer under acidic conditions. The extent of oxidation of immobilized thiol groups via thiol $(-\mathrm{SH}) /$ disulfide (-S-S-) exchange reactions was found to be greater at physiological $\mathrm{pH}$ due to thiolate anion (RS-) formation at high $\mathrm{pH}$. Therefore, these findings support the use of modified chitosan nanoparticles for sustained and controlled release of drug payloads at intracellular infection sites.

\begin{tabular}{|c|c|c|c|c|}
\hline \multirow[t]{2}{*}{ Formulations } & \multicolumn{3}{|c|}{ Uptake studies ( $\mu \mathrm{g} \mathrm{AmB} / 10^{6}$ cells) } & \multirow[t]{2}{*}{ p-value } \\
\hline & Uninfected macrophages & $\begin{array}{l}\text { Sensitive strains-infected } \\
\text { macrophages }\end{array}$ & $\begin{array}{l}\text { Resistant strains- } \\
\text { infected macrophages }\end{array}$ & \\
\hline$A m B$ & $0.4 \pm 0.01$ & $0.38 \pm 0.02$ & $0.04 \pm 0.006$ & $\begin{array}{l}0.058^{x} \\
0.0002^{y} \\
0.0003^{z}\end{array}$ \\
\hline AmBisome & $1.1 \pm 0.07$ & $1.2 \pm 0.08$ & $0.3 \pm 0.05$ & $\begin{array}{l}0.061^{x} \\
0.007^{y} \\
0.0073^{z}\end{array}$ \\
\hline UC & $6.5 \pm 0.9$ & $6.3 \pm 0.7$ & $2.9 \pm 0.4$ & $\begin{array}{l}0.055^{x} \\
0.0004^{y} \\
0.0003^{z}\end{array}$ \\
\hline $\mathrm{TC}$ & $12.9 \pm 2.3$ & $11.5 \pm 1.1$ & $12.3 \pm 2.2$ & $\begin{array}{l}0.056^{x} \\
0.071^{y} \\
0.069^{z}\end{array}$ \\
\hline MTC & $28.6 \pm 1.4^{+* * *, \neq * *, \varsigma^{*}, \eta *}$ & $27.9 \pm 1.6^{\dagger * * *,+* *, \S^{* *}, \eta^{*}}$ & $28.1 \pm 1.7^{\left.\dagger * * *, \neq * *, \zeta^{* *}, 9\right]^{*}}$ & $\begin{array}{l}0.059^{x} \\
0.063^{y} \\
0.064^{z}\end{array}$ \\
\hline \multicolumn{5}{|c|}{ Pretreatment with verapamil } \\
\hline$A m B$ & $0.39 \pm 0.02$ & $0.36 \pm 0.03$ & $0.084 \pm 0.01$ & $\begin{array}{l}0.061^{x} \\
0.003^{y} \\
0.004^{z}\end{array}$ \\
\hline AmBisome & $1.2 \pm 0.06$ & $1.3 \pm 0.09$ & $0.76 \pm 0.05$ & $\begin{array}{l}0.079^{x} \\
0.009^{y} \\
0.008^{z}\end{array}$ \\
\hline UC & $6.6 \pm 0.8$ & $6.2 \pm 0.5$ & $3.5 \pm 0.9$ & $\begin{array}{l}0.062^{x} \\
0.004^{y} \\
0.003^{z}\end{array}$ \\
\hline \multicolumn{5}{|c|}{$\begin{array}{l}\text { Both resistant and sensitive Leishmania donovani were used to infect macrophages. Data are expressed as three replicates in all tests, and } \\
\text { the data are expressed as mean } \pm \text { SD of three independent experiments. Statistically significant differences of MTC were determined in } \\
\left.\text { relation to AmB solution }\left(^{(}\right) \text {, AmBisome }\left(^{*}\right), U C{ }^{(}\right) \text {and TC }\left({ }^{(}\right) \text {at significance levels of }{ }^{*} p<0.05 ;{ }^{* *} p<0.01 ;{ }^{* *} p<0.001 . x=\text { probability } \\
\text { value between uninfected macrophage group and sensitive strain-infected macrophage group; } y=\text { probability value between uninfected } \\
\text { macrophage group and resistant strain-infected macrophage group; } z=\text { probability value between sensitive strain-infected macrophage } \\
\text { group and resistant strain-infected macrophage group. } \\
\text { AmB: Amphotericin B; MTC: Mannose-anchored thiolated chitosan; SD: Standard deviation; TC: Thiolated chitosan; UC: Unmodified chitosan }\end{array}$} \\
\hline
\end{tabular}


The biocompatibility induced by nanoformulations and native drug was assessed at various concentration $(0,25,50$ and $75 \mu \mathrm{g} / \mathrm{ml} \mathrm{AmB})$ in $\mathrm{J} 774$ cells by using the MTT cell proliferation assay. The rank order of biocompatibility at highest concentration was $\mathrm{MTC}>\mathrm{TC}>\mathrm{UC}>\mathrm{AmBisome}>\mathrm{AmB}$. Higher biocompatibility of thiolated nanoformulations (TC and MTC) could be due to the surface modification, where less ionizable-free amino groups are available in TC and MTC as compared with UC. Moreover, surfacemodified nanocarriers resulted in an enormously significant decline $(\mathrm{p} \leq 0.05)$ in the cytotoxicity of AmB. Our findings are similar to those previously reported where surface-modified nanocarriers were used to deliver AmB [2]. The toxicity of AmB has been associated with the formation of drug aggregates [2]. It has been demonstrated that the nonaggregated form of AmB is biocompatible toward mammalian cells but causes porosity of parasitic cells. The aggregated form of $\mathrm{AmB}$ forms pores in both mammalian and parasitic cell membranes. The decline in cytotoxicity of AmB encapsulated into surface-modified nanocarriers could be linked, in measure, to the slow release of nonaggregated drug.

Since macrophages serve as reservoirs for Leishmania amastigotes, receptor-mediated endocytosis of drug nanocarriers is essential in ensuring the delivery of high intracellular drug concentrations required to clear the parasite. We therefore sought to design and study MTC nanocarriers for cellular internalization of antileishmanial agents. The cellular internalization of nanoformulations and native drug was compared in sensitive and resistant $L$. donovani-infected or -uninfected control cells. Overexpression of ABC transporters especially MDR1 is well known to be an important mechanism of drug efflux for $L$. donovaniresistant strains [18]. Hence, lower cellular internalization of UC nanocarriers, AmBisome and native drug into cells infected with the resistant VL strain could be due to upregulated drug efflux machinery. In contrast, no significant difference in cellular internalization profile of TC and MTC was seen in sensitive and resistant $L$. donovani-infected or -uninfected control cells. Efflux pump inhibitory capability of thiolated polymers has been shown in mammalian cells [11]. Dünnhaupt et al. demonstrated that thiolated polymers inhibit the efflux pump by blocking the efflux pump drug-binding sites [22]. Accordingly, TC and MTC may be also responsible for the inhibition of drug efflux machinery in the resistant VL strain.

Thiol-reactive groups have also been shown to promote binding and cellular internalization of nanomaterials $[13,23,24]$. A recent study on thiolated surfacestabilized superparamagnetic iron oxide nanoparticles

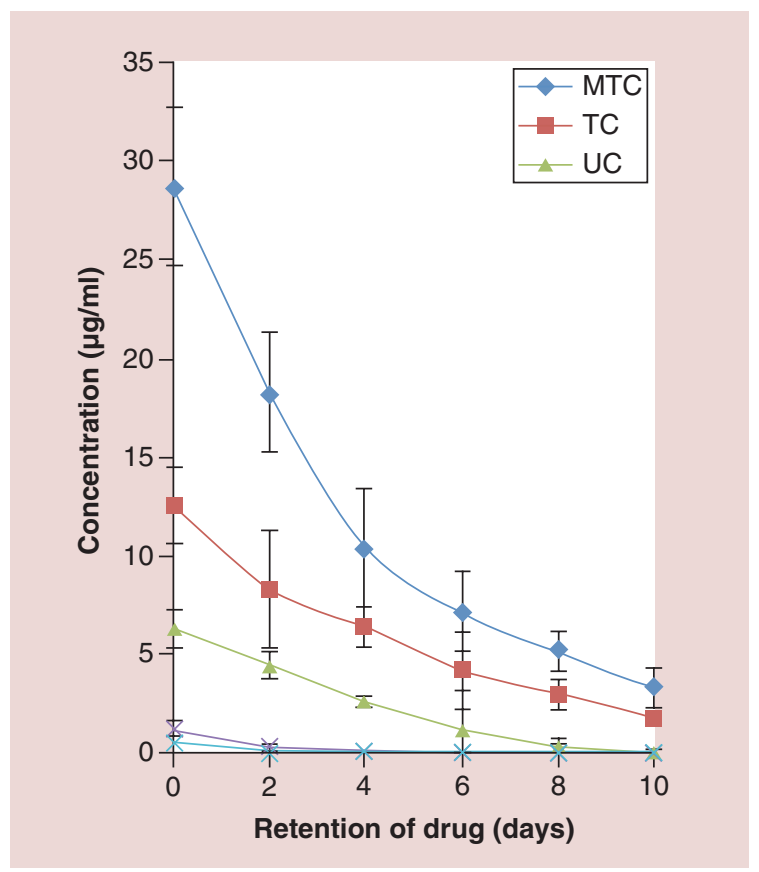

Figure 7. Intracellular retention of MTC, TC, UC nanocarriers, AmBisome ${ }^{\oplus}$ and native drug in macrophages determined over 10 days. Retention of drug was observed along with sustained release. Data are shown as mean \pm SD of three replicate wells. $* * * p<0.001$ (comparison of MTC versus AmB and AmBisome); ${ }^{* *} p<0.01$ (comparison of MTC versus TC and UC nanocarriers).

AmB: Amphotericin B; MTC: Mannose-anchored thiolated chitosan; SD: Standard deviation;

TC: Thiolated chitosan; UC: Unmodified chitosan.

demonstrated efficient internalization of thiol surfacemodified nanoparticles by stem cells. It was found that decorating the magnetic particles with thiol moieties resulted in a 17-fold increase in uptake by progenitor cells when compared with nonthiolated superparamagnetic iron oxide nanoparticles [20]. These findings provide additional support to the concept that the presence of thiol groups on the surface of the particles could enhance cellular internalization. Accordingly, a 1.98-fold increase in uptake of TC by noninfected macrophages was observed over equivalent concentrations of UC nanocarriers. Further improvement in AmB cellular uptake was seen when MTC was used as the drug carrier. The increase in uptake for the mannose-anchored MTC over the TC may be due to the mannose receptor-mediated endocytosis of the drug particles. The macrophage-targeted MTC drug delivery system therefore represents a promising approach to Leishmania eradication that would allow for delivery of effective drug concentrations at sites of amastigote replication. Efficient internalization of MTC into macrophages may proceed by either specific or nonspecific mechanisms that include: access and initial interaction 


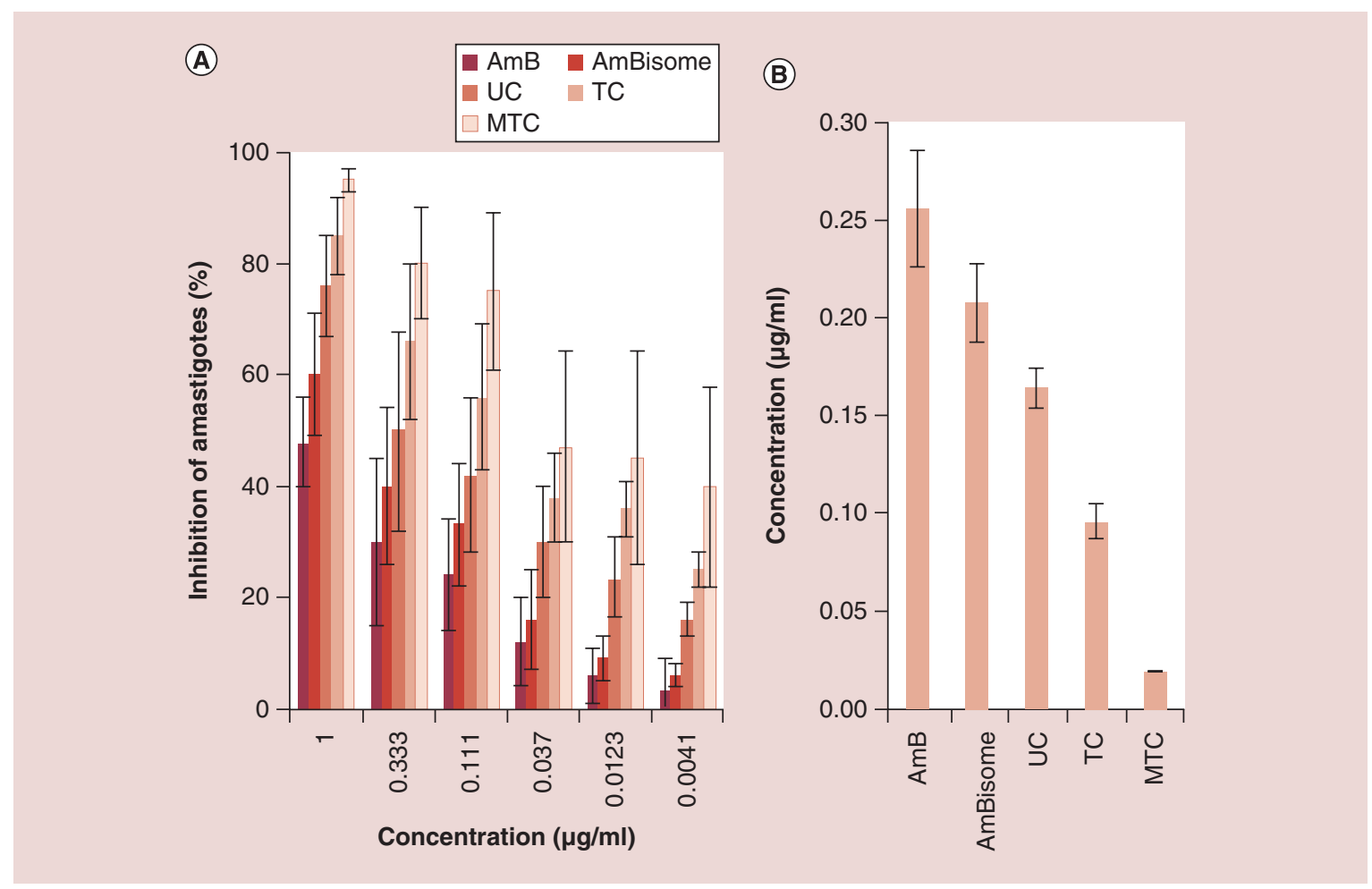

Figure 8. Pharmacological evaluation of antileishmanial activities. Percent inhibition of Leishmania donovani amastigotes by escalating concentration ( $\mathrm{ug} / \mathrm{ml}$ ) of AmB, AmBisome, UC, TC and MTC (A). For

(B) the concentration $(\mathrm{ug} / \mathrm{ml})$ required to achieve an in vitro $\left(\mathrm{IC}_{50}\right)$ activity against $L$. donovani amastigotes. All experiments were performed with human MDM infected with the parasite for a $12 \mathrm{~h}$ incubation (B). Concentration of the polymer used in nanoformulation was biocompatible to the target macrophage. Data expressed as mean \pm SD for a representative of one or three performed experiments.

AmB: Amphotericin B; MDM: Monocyte-derived macrophages; MTC: Mannose-anchored thiolated chitosan; SD: Standard deviation; UC: Unmodified chitosan.

of nanocarriers with the surface membrane of macrophages; interaction of reactive thiol moieties on the surface of MTC with the macrophage membrane; recognition of MTC by mannose phagocytic machinery expressed on the macrophage surface; and configurational changes in the cell membrane structure (projection or invagination), vacuolization and internalization of the drug particles. Therefore, increased intracellular drug concentrations and prolonged retention exhibited by MTC are all important aspects in the development of nanoformulations that would target and eradicate intracellular infections.

Superior cellular retention profiles of the UC, TC and MTC nanocarriers as compared with AmBisome and native drug could be due to cationic chitosan polymeric backbone. Chitosan-based nanocarriers are known to escape from the endosomes thereby able to escape lysosomal degradation and deliver the drug payload to the target site [25]. This phenomenon that is common to cationic polymers is referred to a proton sponge effect [26]. In addition, the extended sustained and slow release of drug for TC and MTC nanocarriers might be the result of interactions between thiol moi- eties of the nanocarriers and the disulfide bonds of the lysosomal enzyme via thiol/disulfide exchange reaction $(\mathrm{SH} \rightarrow-\mathrm{S}-\mathrm{S}-;$-S-S- $\rightarrow-\mathrm{SH})$. This prolonged retention profile would allow for a reduced dosing frequency.

Leishmania parasites have two main life-cycle phases: the extracellular motile promastigotes phase, found in the phlebotomine sandflies vector and the intracellular immotile amastigotes phase that thrives in the mammalian host. Amastigotes proliferate in the macrophage parasitophorous vacuoles, phagolysosome-like compartments with hydrolytic and acidic surroundings. Parasitophorous amastigotes lead to destruction of the host cell but manage to escape into the systemic circulation where they are adept at infecting new phagocytic cells and responsible for the pathogenesis of leishmaniasis. Targeted drug delivery to parasite reservoirs such as macrophages is anticipated to increase the therapeutic efficacy and diminish off-target drug toxicity. For antileishmanial activity against parasitophorous $L$. donovani amastigotes-infected macrophages, MTC again provided maximum inhibition of the parasite. Low drug concentrations of the MTC formulation were able to inhibit intracellular 
parasite replication when compared with the clinically used AmBisome formulation ( $\mathrm{p}<0.05)$. The $\mathrm{IC}_{50}$ of MTC was 13.0-, 10.6-, 8.4- and 4.9-fold lower than that of AmB ( $p<0.05)$, AmBisome $(\mathrm{p}<0.05)$, Chito $(\mathrm{p}<0.05)$ and CT $(\mathrm{p}<0.05)$, respectively. The observed improvement in antileishmanial activity of the MTC AmB nanocarrier could be the result of localized delivery of the therapeutic agent at intracellular sites that harbor the parasites.

Oral MTC nanoformulation was highly efficacious in infected rodents. These results support our laboratory findings of enhanced antimicrobial activities of the nanoformulations. The efficacy of the MTC nanoformulations was significantly greater than that of the AmB solution. This improved efficacy may be associated with an improved cellular internalization of the drug formulations with a favored accumulation in the reticuloendothelial system.

We found drug-free MTC control nanoformulations to exhibit antileishmanial activity. Mannosylated nanocarriers have been shown to generate signals following engulfment or interaction with phagocytic cells, in particular macrophages, which leads to macrophage stimulation and upregulation of cytokines [17]. Inducible nitric oxide synthase and consecutive generation of nitric oxide has been shown to exhibit antimicrobial effect [27]. The chitosan polymer has been reported to trigger a proinflammatory response such as nitric oxide production that stimulates macrophages and this could lead to parasiticidal activity [17,27]. Hence, antileishmanial activity of drug-free nanocarriers based on chitosan polymeric backbone could be due to nitric oxide production. Therefore, higher drug uptake and successful killing of $L$. donovani parasites by the MTC formulation are expected to attain the preferred therapeutic outcome at a lower dose.

\section{Conclusion}

Long acting macrophage-targeted MTC nanocarriers loaded with $A \mathrm{mB}$ were successfully synthesized and evaluated for cellular uptake and antileishmanial responses. The mannosylated nanocarriers displayed enhanced uptake of the encapsulated AmB by macrophages when compared with uptake of native drug or nontargeted particles. Interestingly, the mannosylated system was found to retain the drug inside the macrophages for up to 10 days. The MTC nanocarriers were also found to be highly effective at inhibiting the growth of intracellular amastigotes in infected macrophages when compared with native drug. These encouraging in vitro data provide proof-of-concept that macrophagedirected TC nanocarriers hold promise as potential therapies for parasitic and other intracellular infections.

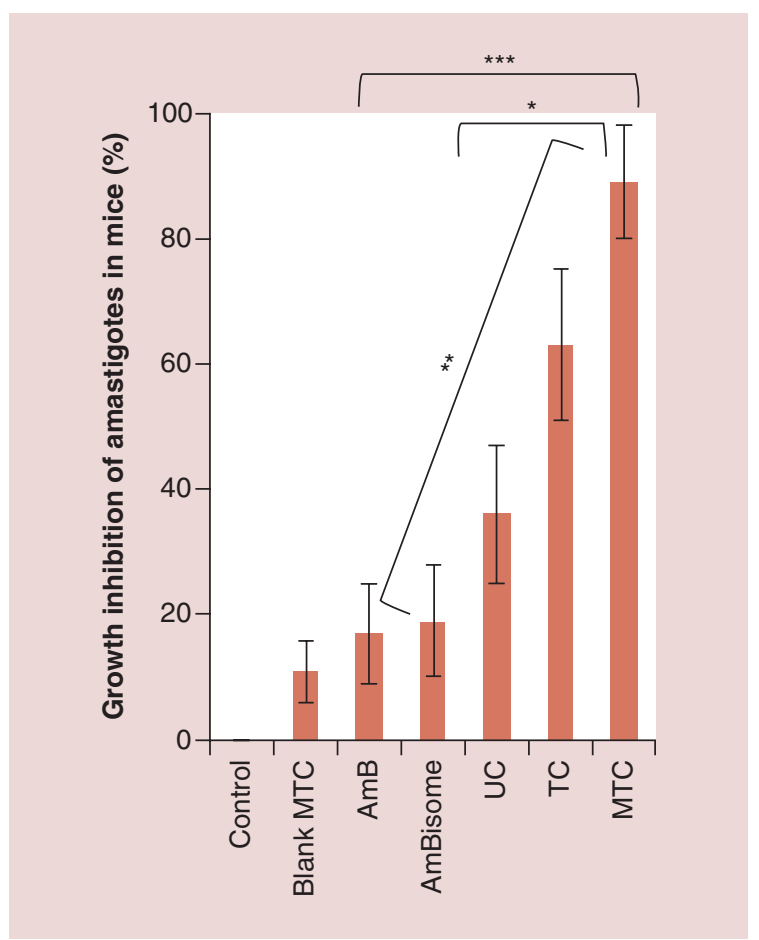

Figure 9. In vivo therapeutic efficacy of oral MTC, TC, UC, AmB and blank MTC nanocarriers (AmB free) in BALB/c mice infected with Leishmania donovani at an oral dose of $1 \mathrm{mg}$ of $\mathrm{AmB} / \mathrm{kg}$ body weight. AmBisome ${ }^{\circledR}$ was injected intravenously, as an additional control, into mice on the 4th week of infection. Data are expressed as median values \pm SD and significant differences were determined by Kruskal-Wallis test, followed by Dunn's posthoc test with significance levels ${ }^{*} p<0.05 ;{ }^{* *} p<0.01 ; * * * p<0.001$.

AmB: Amphotericin B; MTC: Mannose-anchored thiolated chitosan; SD: Standard deviation;

TC: Thiolated chitosan; UC: Unmodified chitosan.

\section{Future perspective}

$\mathrm{AmB}$ for VL is currently administered by parenteral route because of poor oral bioavailability. But high cost and associated nephrotoxicity have restricted parenteral administration of AmB. Therefore, development of an oral formulation of AmB for VL will help overcome these limitations. The synthesized mannosylated and TC delivery system with permeation enhancing, in situ gelling, sustained release, mucoadhesion and P-glycoprotein inhibitory properties has the potential to improve oral bioavailability and efficacy of AmB.

\section{Acknowledgements}

The authors thank KP Chang and S Rana for technical assistance and active research discussions. The authors also acknowledge the assistance of the University of Nebraska Lincoln Electron Microscopy Core Facility. 
Financial \& competing interests disclosure

This work was supported by the UNESCO-L'Oréal International Fellowship for Young Women in Life Sciences and $\mathrm{NIH}$ grants P01 DA028555, R01 NS36126, P01 NS31492, 2R01 NS034239, P01 MH64570, P01 NS43985, P30 MH062261 and R01 AG043540. The authors have no other relevant affiliations or financial involvement with any organization or entity with a financial interest in or financial conflict with the subject matter or materials discussed in the manuscript apart from those disclosed.

No writing assistance was utilized in the production of this manuscript.
Ethical conduct of research

The authors state that they have obtained appropriate institutional review board approval or have followed the principles outlined in the Declaration of Helsinki for all human or animal experimental investigations. In addition, for investigations involving human subjects, informed consent has been obtained from the participants involved.

\section{Open access}

This work is licensed under the Creative Commons Attribution-NonCommercial 4.0 Unported License. To view a copy of this license, visit http://creativecommons.org/licenses/by-nc$\mathrm{nd} / 4.0$ /

\section{Executive summary}

- Mannose-anchored thiolated chitosan (MTC) nanocarriers were designed and evaluated against intramacrophage amastigote parasites.

- Surface-modified nanocarriers resulted in an enormously significant decline in the cytotoxicity of amphotericin B.

- Thiolated chitosan and MTC nanocarriers were rapidly internalized into the macrophages and contributed to inhibition of amphotericin B efflux in resistant strain-infected macrophages.

- At lower concentrations, the MTC formulation were able to inhibit intracellular parasite replication as compared with the AmBisome ${ }^{\circledR}$.

- Finally, MTC nanocarriers emerge to be a promising nanomedical tool for the treatment of leishmaniasis.

\section{References}

Papers of special note have been highlighted as: $\bullet$ of interest; •• of considerable interest

1 Yasinzai M, Khan M, Nadhman A, Shahnaz G. Drug resistance in leishmaniasis: current drug-delivery systems and future perspectives. Future Med. Chem. 5(15), 1877-1888 (2013).

- Expert opinion on therapeutic strategies for visceral leishmaniasis.

2 Jain K, Verma AK, Mishra PR, Jain NK. Surface-engineered dendrimeric nanoconjugates for macrophage-targeted delivery of amphotericin B: formulation development and in vitro and in vivo evaluation. Antimicrob. Agents Chemother. 59(5), 2479-2487 (2015).

3 Asthana S, Gupta PK, Jaiswal AK, Dube A, Chourasia MK. Overexpressed macrophage mannose receptor targeted nanocapsules-mediated cargo delivery approach for eradication of resident parasite: in vitro and in vivo studies. Pharm. Res. 32(8), 2663-2677 (2015).

-• Describes macrophage mannose receptor-targeted antileishmanial therapy.

4 Jain K, Verma AK, Mishra PR, Jain NK. Characterization and evaluation of amphotericin B loaded MDP conjugated poly(propylene imine) dendrimers. Nanomedicine 11(3), 705-713 (2015).

5 Chaubey P, Mishra B. Mannose-conjugated chitosan nanoparticles loaded with rifampicin for the treatment of visceral leishmaniasis. Carbohydr. Polym. 101, 1101-1108 (2014).
6 Dutta T, Agashe HB, Garg M, Balakrishnan P, Kabra M, Jain NK. Poly (propyleneimine) dendrimer based nanocontainers for targeting of efavirenz to human monocytes/macrophages in vitro. J. Drug Target. 15(1), 89-98 (2007).

7 Sarkar K, Das PK. Protective effect of neoglycoproteinconjugated muramyl dipeptide against Leishmania donovani infection: the role of cytokines. J. Immunol. 158(11), 5357-5365 (1997).

8 Jain NK, Mishra V, Mehra NK. Targeted drug delivery to macrophages. Expert Opin. Drug Deliv. 10(3), 353-367 (2013).

9 Yeeprae W, Kawakami S, Higuchi Y, Yamashita F, Hashida $\mathrm{M}$. Biodistribution characteristics of mannosylated and fucosylated $\mathrm{O} / \mathrm{W}$ emulsions in mice. J. Drug Target. 13(8-9), 479-487 (2005).

- Describes the synthesis and characterization of thiolated polymers.

10 Kast CE, Bernkop-Schnurch A. Thiolated polymers-thiomers: development and in vitro evaluation of chitosan-thioglycolic acid conjugates. Biomaterials 22(17), 2345-2352 (2001).

- Describes the synthesis of nanoparticles followed by in vitro and in vivo studies.

11 Palmberger TF, Hombach J, Bernkop-Schnurch A. Thiolated chitosan: development and in vitro evaluation of an oral delivery system for acyclovir. Int. J. Pharm. 348(1-2), 54-60 (2008).

12 Shahnaz G, Vetter A, Barthelmes J et al. Thiolated chitosan nanoparticles for the nasal administration of leuprolide: bioavailability and pharmacokinetic characterization. Int. J. Pharm. 428(1-2), 164-170 (2012). 
13 Vetter A, Reinisch A, Strunk D et al. Thiolated polyacrylic acid-modified iron oxide nanoparticles for in vitro labeling and MRI of stem cells. J. Drug Target. 19(7), 562-572 (2011).

14 Veerareddy PR, Vobalaboina V, Ali N. Antileishmanial activity, pharmacokinetics and tissue distribution studies of mannose-grafted amphotericin B lipid nanospheres. J. Drug Target. 17(2), 140-147 (2009).

15 Yalpani M, Laurance DH. Some chemical and analytical aspects of polysaccharide modifications 3 . Formation of branch-chain, soluble chitosan derivatives. Macromolecules 17, 272-281 (1984).

16 Javed I, Hussain SZ, Ullah I et al. Synthesis, characterization and evaluation of lecithin-based nanocarriers for the enhanced pharmacological and oral pharmacokinetic profile of amphotericin B. J. Mater. Chem. B 3(42), 8359-8365 (2015).

17 Asthana S, Jaiswal AK, Gupta PK, Pawar VK, Dube A, Chourasia MK. Immunoadjuvant chemotherapy of visceral leishmaniasis in hamsters using amphotericin B-encapsulated nanoemulsion template-based chitosan nanocapsules. Antimicrob. Agents Chemother. 57(4), 1714-1722 (2013).

Purkait B, Kumar A, Nandi N et al. Mechanism of amphotericin B resistance in clinical isolates of Leishmania donovani. Antimicrob. Agents Chemother. 56(2), 1031-1041 (2012).

19 Zhou W, Wang Y, Jian J, Song S. Self-aggregated nanoparticles based on amphiphilic poly(lactic acid)-graftedchitosan copolymer for ocular delivery of amphotericin B. Int. J. Nanomedicine 8, 3715-3728 (2013).

20 Shahnaz G, Kremser C, Reinisch A et al. Efficient MRI labeling of endothelial progenitor cells: design of thiolated surface stabilized superparamagnetic iron oxide nanoparticles. Eur. J. Pharm. Biopharm. 85 (3 Pt A), 346-355 (2013).
21 Shahnaz G, Perera G, Sakloetsakun D, Rahmat D, BernkopSchnurch A. Synthesis, characterization, mucoadhesion and biocompatibility of thiolated carboxymethyl dextran-cysteine conjugate. J. Control. Release 144(1), 32-38 (2010).

- Comprehensive study on thiomers as efflux pump inhibitors.

22 Dunnhaupt S, Barthelmes J, Rahmat D et al. S-protected thiolated chitosan for oral delivery of hydrophilic macromolecules: evaluation of permeation enhancing and efflux pump inhibitory properties. Mol. Pharm. 9(5), 1331-1341 (2012).

-. Explains the mechanism for cellular internalization of surface thiol groups.

23 Aubry S, Burlina F, Dupont E et al. Cell-surface thiols affect cell entry of disulfide-conjugated peptides. FASEB J. 23(9), 2956-2967 (2009).

24 Rahmat D, Khan MI, Shahnaz G, Sakloetsakun D, Perera G, Bernkop-Schnurch A. Synergistic effects of conjugating cell penetrating peptides and thiomers on non-viral transfection efficiency. Biomaterials 33(7), 2321-2326 (2012).

25 Agirre M, Zarate J, Ojeda E, Puras G, Desbrieres J, Pedraz JL. Low molecular weight chitosan (LMWC)-based polyplexes for pDNA delivery: from bench to bedside. Polymers (Basel, Switz.) 6(6), 1727-1755, 1729 (2014).

26 Malatesta M, Grecchi S, Chiesa E, Cisterna B, Costanzo M, Zancanaro C. Internalized chitosan nanoparticles persist for long time in cultured cells. Eur. J. Histochem. 59(1), 2492 (2015).

27 Gupta PK, Jaiswal AK, Asthana S et al. Self assembled ionically sodium alginate cross-linked amphotericin B encapsulated glycol chitosan stearate nanoparticles: applicability in better chemotherapy and non-toxic delivery in visceral leishmaniasis. Pharm. Res. 32(5), 1727-1740 (2015). 